\title{
WORST OUTCOME IN SCLERODERMA RENAL CRISIS ASSOCIATED WITH COVID-19 NEPHROPATHY
}

Caroline Pamponet da Fonseca Oliveira ${ }^{1, \star}$, Gabriela Cruz de Oliveira ${ }^{1}$, Junior Apaestegui Coriat ${ }^{1}$, Camila Pamponet da Fonseca

Oliveira² ${ }^{2}$ Isabelle Santos Alves ${ }^{2}$, Anne Caroline da Silva Menezes ${ }^{1}$, Bárbara Seabra Carneiro ${ }^{1}$

1 Hospital Universitário Getúlio Vargas, Manaus (AM), Brazil. 2 Universidade Federal do Amazonas, Manaus (AM), Brazil

*Corresponding author: carolinepamponetfo@gmail.com

\section{BACKGROUND}

Systemic Sclerosis (SSc) is an autoimmune disease which may evolve with severe renal impairment. The scleroderma renal crisis leads to arterial hypertension and renal dysfunction. It is a potentially fatal complication that occurs in approximately $10 \%$ of patients. Severe forms of COVID-19 present renal involvement in 20 to $40 \%$ of patients who require intensive care. There are few reports of this infection in patients with systemic sclerosis.

\section{CASE REPORT}

AXL, female, 29 years old, with overlapping SSc and systemic lupus erythematosus for 11 years, under use of prednisone 40mg, azathioprine $100 \mathrm{mg}$ and chloroquine $150 \mathrm{mg}$ daily. The patient started flu-like symptoms, presenting with cough, fever, chest pain and dyspnea. After one week, she developed respiratory distress and desaturation, requiring orotracheal intubation and ICU management. She also had edema in the lower limbs, livedo reticularis and Raynaud's phenomenon in hands. The chest tomography demonstrated diffuse ground-glass opacities bilaterally, compromising $50 \%$ of the lung parenchyma, in addition to mild pleural and pericardial effusion. Echocardiogram showed diffuse left ventricular hypokinesia. Detectable PCR for SARS-CoV-2 confirmed the new coronavirus infection. The patient underwent treatment with methylprednisolone $62.5 \mathrm{mg}$ daily and piperacillin with tazobactam. During hospitalization, she developed hypertensive conditions, macroscopic hematuria and acute renal failure due to a probable scleroderma renal crisis. Laboratory tests showed renal dysfunction (creatinine of $3.4 \mathrm{mg} / \mathrm{dl}$, urea of $170 \mathrm{mg} / \mathrm{dll}$ ), microangiopathic hemolytic anemia (hemoglobin of $8.6 \mathrm{~g} / \mathrm{dl}$, hematocrit of $29.2 \%$ ), elevation of liver enzymes (TGO $744 \mathrm{U} / \mathrm{l}, \mathrm{TGP} 452 \mathrm{U} / \mathrm{l}$ ), proteinuria of 532mg in 24 hours, low C3 and metabolic acidosis. Initially, a treatment with captopril 300mg daily was instituted, but dialysis therapy was needed. The patient evolved gravely, with septic shock, no improvement under use of antibiotic therapy, antifungal and vasoactive drugs. She passed away after 1 month of hospitalization.

\section{CONCLUSION}

SARS-CoV-2 kidney failure has a multifactorial etiology, which may occur due to direct viral endothelial and epithelial injury, cardiorenal syndrome or sepsis. COVID-19 renal and cardiovascular complications, corticosteroid use in inflammatory phase of the infection and renal impairment of underlying autoimmune diseases, possibly contributed to this unfavorable progression of renal dysfunction in the scleroderma crisis. 